\title{
Cuidando de quem cuida: Avaliação da Neuropatia e Prevalência do Diabetes Mellitus 2
}

\author{
Caring for those who care: Assessment of Neuropathy and Prevalence of Diabetes Mellitus 2 \\ Cuidando a los que cuidan: Evaluación de la neuropatía y la prevalencia de la Diabetes Mellitus 2
}

\begin{abstract}
RESUMO
Objetivo: Identificar a prevalência do diabetes mellitus 2 (DM2) em cuidadores e inspecionar os pés em diabéticos autorreferidos. Método: Estudo quantitativo transversal, amostragem probabilística de conveniência em cuidadores de crianças especiais atendidas em uma instituição filantrópica com a aplicação de um questionário direto, em diabéticos autorreferidos pelo questionário ofertou-se a inspeção dos pés seguindo o "Formulário para Avaliação de Neuropatia e Doença Arterial Periférica - Ambulatório de Especialidade" com os instrumentos: Mono lamento de Semmes-Weinstein de 10g, diapasão 128Hz, martelo de re exo. Resultados: Verificou-se a prevalência de 23,7\% diabéticos no público de 114 entrevistados. Na inspeção identificou-se: pele ressecada, fissuras, micose, cor de pele alterada, vasos dilatados dorsais e edemas. Conclusão: Observou-se a problemática de uma realidade local em uma instituição filantrópica, os dados não são generalizáveis. Evidencia-se a necessidade de estratégias de rastreamento e intervenção com foco na atenção primária e autocuidado para evitar amputações e outros agravos decorrentes DM2.
\end{abstract}

DESCRITORES: Neuropatias Diabéticas; Diabetes Mellitus; Prevalência.

\section{ABSTRACT}

Objective: To identify the prevalence of diabetes mellitus 2 (DM2) in caregivers and inspect the feet in self-reported diabetics. Method: Quantitative cross-sectional study, probabilistic sampling of convenience in caregivers of special children cared for in a philanthropic institution with the application of a direct questionnaire, in diabetics self-reported by the questionnaire, offered the inspection of the feet following the "Form for Assessment of Neuropathy and Disease Peripheral Arterial - Specialty Clinic "with the instruments: $10 \mathrm{~g}$ Semmes-Weinstein monofilament, $128 \mathrm{~Hz}$ tuning fork, reflective hammer. Results: There was a prevalence of $23.7 \%$ diabetics in the public of 114 respondents. During inspection, it was identified: dry skin, fissures, ringworm, altered skin color, dilated dorsal vessels and edema. Conclusion: We observed the problem of a local reality in a philanthropic institution, the data are not generalizable. The need for screening and intervention strategies focusing on primary care and self-care is evident in order to avoid amputations and other injuries resulting from DM2.

DESCRIPTORS: Diabetic Neuropathies; Diabetes Mellitus; Prevalence.

\section{RESUMEN}

Objetivo: Identificar la prevalencia de diabetes mellitus 2 (DM2) en cuidadores e inspeccionar los pies en diabéticos autonotificados. Método: Estudio cuantitativo transversal, muestreo probabilístico de conveniencia en cuidadores de niños especiales atendidos en una institución filantrópica con la aplicación de un cuestionario directo, en diabéticos autoinformados por el cuestionario, ofrecido la inspección de los pies siguiendo el "Formulario para Evaluación de Neuropatía y Enfermedad Arterial Periférica - Clínica de Especialidades "con los instrumentos: monofilamento Semmes-Weinstein de 10g, diapasón de 128Hz, martillo reflectante. Resultados: Hubo una prevalencia del 23,7\% de diabéticos en el público de 114 encuestados. Durante la inspección se identificó: piel seca, fisuras, tiña, alteración del color de la piel, vasos dorsales dilatados y edema. Conclusión: Observamos el problema de una realidad local en una institución filantrópica, los datos no son generalizables. Es evidente la necesidad de estrategias de cribado e intervención centradas en la atención primaria y el autocuidado para evitar amputaciones y otros problemas derivados de la DM2.

DESCRIPTORES: Neuropatías Diabéticas; Diabetes Mellitus; Prevalencia.

RECEBIDO EM: 19/03/2021 APROVADO EM: 13/04/2021

\section{Thalia de Lima Sousa}

Graduanda de Enfermagem. Universidade Paulista UNIP. Santana de Parnaíba, São Paulo.

ORCID: 0000-0003-0207-8664 


\section{Claudia Neves Nascimento da Costa}

Enfermeira. Pós-graduanda em Enfermagem Obstétrica e Ginecológica no Instituto Israelita de Ensino e Pesquisa Albert Einstein. ORCID: 0000-0002-5490-4633

\section{INTRODUÇÃO}

0 Diabetes Mellitus é uma doença crônica metabólica que tem por característica principal o aumento dos níveis glicêmicos, na qual pode ser causada devido defeitos relacionados a ação e/ ou secreção da insulina ${ }^{1}$. As classificações atuais são o tipo 1 , tipo 2 , gestacional e outros tipos específicos de diabetes mellitus. No Diabetes Mellitus tipo 2 (DM2) há a resistência à insulina que é quando o organismo produz a insulina, porém as células não a utilizam adequadamente pois estão com suas ações diminuídas e não são capazes de realizar de maneira efetiva a hipoglicemia, que colabora com o aumento da glicemia e associa ao aumento da insulina sanguínea. Quando em períodos prolongados deste quadro, poderá haver danos em órgãos, vasos sanguíneos e nervos ${ }^{1}$. Diversos fatores de riscos podem contribuir para o acometimento da DM2, como alimentação inadequada, sedentarismo, presença de parentes de $1^{\circ}$ e $2^{\circ}$ grau, entre outros fatores ${ }^{2}$. Dentre as complicações do diabetes, ressalta-se a neuropatia diabética que acomete cerca de $70 \%$ dos pacientes portadores de DM2, este quadro é progressivo e severo que pode levar a ulceração e a amputação, além de causar parestesia dolorosa, ataxia sensorial e o pé de Charcot ${ }^{3}$.

Wilson ${ }^{4}$ define o cuidador como um indivíduo que assume as responsabilidades essenciais do paciente, podendo este ser familiar ou amigo. Estudos demonstram que o cuidador abdica do autocuidado para cuidar do assistido5, Dorothea Orem $^{6}$ descreve o autocuidado como a função humana que indivíduos realizam para sanar as necessidades essenciais a vida e a integridade da sua própria função e desenvolvimento ${ }^{7}$. No âmbito de Crianças com Necessidades Especiais de Saúde (CRIANES), os diferentes agravos e complexidade dos cuidados requerem maior atenção e acompanhamento dos serviços
O Diabetes Mellitus

é uma doença crônica metabólica

que tem por

característica

principal o

aumento dos níveis

glicêmicos,

na qual pode ser

causada devido

defeitos relacionados

a ação e/ou secreção

da insulina.

As classificações

atuais são o tipo 1,

tipo 2, gestacional

e outros tipos

específicos de

diabetes mellitus. de saúde por parte do cuidador, cujo indivíduo que poderá ter a própria qualidade de vida diminuída pela dispensação de cuidados frequentes a si próprio ${ }^{8}$.

A prevalência mundial do diabetes mellitus é alarmante, sendo apontada como a quarta principal causa de morte e uma das doenças crônicas não transmissíveis de maior acometimento9. Conforme dados nacionais da SBD em 2019 ${ }^{10}$, a doença acomete 16,8 milhões de pessoas, representando $7,9 \%$ da população brasileira e prevê o aumento para 49 milhões em 2045. Em caso de demora para o diagnóstico, seja por procura tardia do serviço de saúde ou atraso no atendimento, há o favorecimento de maiores complicações do DM21 No diagnóstico precoce há a oportunidade essencial da realização de prevenção de agravos, o paciente poderá buscar e implementar cuidados específicos antes que a morbidade se torne significante ${ }^{11}$.

No Brasil, o Sistema Único de Saúde (SUS) tem como porta de entrada para o atendimento de saúde a Atenção Primária de Saúde, onde deverá ocorrer o acolhimento para prevenção e controle do DM2 seguindo os princípios de universalidade, integralidade e equidade das ações públicas. Faz-se necessário rastrear e caracterizar as condições de saúde local para inserir estratégias de prevenção e intervenção conforme as necessidades identificadas, no âmbito individual e coletivo ${ }^{12}$.

Os profissionais de saúde são ferramentas necessárias para enfrentamento da doença, com foco nos Enfermeiros que são preparados para prestar a assistência e auxiliar com medidas preventivas, curativas e educativas ${ }^{13}$. Foram realizados mutirões de saúde em uma instituição filantrópica localizada na região metropolitana de São Paulo e foi observado que os valores de glicemia capilar pós-prandial de algumas cuidadoras, bem como pressão arterial e relação cintura quadril estavam alterados, demonstrando uma possível correlação com sintomas da síndrome metabólica. 
Diante do exposto, foram realizadas duas pesquisas paralelas com as seguintes questões norteadoras: Qual a prevalência do DM2 nos cuidadores de CRIANES atendidas em uma instituição filantrópica localizada na região metropolitana de São Paulo?; Qual a prevalência da predisposição genética para o DM2 nestes cuidadores?; Para cuidadores diabéticos, quais sinais e sintomas podem ser encontrados na inspeção dos pés? Assim, o objetivo deste estudo foi de identificar a prevalência do DM2 e inspecionar os pés de diabéticos confirmados em cuidadores de CRIANES assistidos em uma associação beneficente localizada na região metropolitana de São Paulo.

\section{MÉTODOS}

Estudo quantitativo transversal com amostragem probabilística de conveniência. Neste estudo a coleta de dados da prevalência foi realizada por meio de um questionário para assinalar aplicado durante a visita de rotina na instituição filantrópica em cuidadores de crianças portadoras de deficiências múltiplas após a explicação do que se trata e pesquisa e coleta da assinatura do Termo de Consentimento Livre e Esclarecido, no período de setembro de 2018 a maio de 2019 com intervalo entre dezembro e fevereiro, com as seguintes questôes: Você possui DM2? Há quanto tempo? Tem mais alguma pessoa na sua casa que é diabética(o)? Na sua família tem alguém diabético? Qual o grau de parentesco? Aos cuidadores que confirmaram ser diabéticos do tipo 2 foi oferecido a inspeção dos pés, seguindo o Protocolo de Cuidado com os Pés de Pessoas com Formulário para Avaliação de Neuropatia e Doença Arterial Periférica -Ambulatório de Especialidade preenchido pelo inspecionador com os instrumentos: Monofilamento de Semmes-Weinstein de $10 \mathrm{~g}$, diapasão $128 \mathrm{~Hz}$, martelo de reflexo. ${ }^{14}$

Os critérios de inclusão para a aplicação do questionário foram: no máximo, 2 cuidadores diretos das crianças e adolescentes atendidas na instituição filantrópica, concordar em preencher o questionário e possuir Termo de Consentimento Livre e
Esclarecido (TCLE) devidamente assinado previamente, idade acima de 18 anos. Como critério de exclusão os indivíduos que não possuem interesse em participar

Os critérios de inclusão para a aplicação do questionário foram: no máximo, 2 cuidadores diretos

das crianças e adolescentes atendidas na instituição filantrópica, concordar em preencher $\mathbf{o}$ questionário e possuir Termo de

Consentimento

Livre e Esclarecido (TCLE) devidamente assinado

previamente, idade acima de 18 anos da pesquisa. Para a participação da inspeção nos pés, os critérios de inclusão foram: Afirmar no questionário ser diabético tipo 2, preencher o TCLE previamente a inspeção e estar de acordo com os demais critérios de inclusão da aplicação do questionário. Como critério de exclusão os indivíduos que não possuem interesse em participar da pesquisa.

Pesquisa aprovada pelo Comitê de Ética em Pesquisa da Universidade Paulista que recebe e avalia projetos de pesquisa envolvendo seres humanos, sob o número para aplicação do questionário $\quad 96788618.3 .0000 .5512$ e para inspeção dos pés sob o número 97452918.0.0000.5512. Os autores garantiram o seguimento de todas as definições descritas na resolução $466 / 12^{15}$.

Os dados obtidos foram transcritos para o software Microsoft Excel 2016 e elaborados com auxílio do software Microsoft Word 2016, o conteúdo foi analisado por meio de análise de Bardin ${ }^{16}$ com a estruturação das etapas: análise previa, a primeira etapa que os dados coletado são submetidos a um estudo mais aprofundado; descrição analítica, que é a classificação de cada dado obtido e seus tópicos que cruzam com os objetivos deste; interpretação inferencial, relacionar os resultados obtidos com a realidade estudada.

\section{RESULTADOS}

Foi realizado a aplicação do questionário em 114 cuidadores e a inspeção ocorreu em 27 cuidadores diabéticos. Os resultados obtidos através do questionário foram analisados conforme demonstra Quadro I.

Entre os 23,68\% cuidadores que confirmaram possuir a diabetes mellitus tipo 2 foi realizado um segundo quadro para análise comparativa de gênero e idade da amostra diabética, conforme quadro 2 .

Quanto a avaliação dos pés diabéticos, os 27 portadores da doença consentiram em participar e entre eles $42,9 \%$ relataram dores nas pernas e $57,1 \%$ assintomáticos. $\mathrm{O}$ escore de neuropatia identificado nos cuidadores: escore 1 em 21,4\%, escore 2 
em 7,1\%, escore 3 a 5 em 14\%.

A inspeção dos pés apresentou $48 \%$ pele ressecada e fissuras, $40 \%$ calosidades, $37 \%$ micose ungueal ou digital, $7,4 \%$ cor de pele anormal, $11 \%$ vasos dilatados dorsais, $11 \%$ edemas.

\section{DISCUSSÃO}

Neste estudo, foram apresentados o autorrelato de cuidadores sobre possuírem ou não o diabetes por meio do questionário e foi possível identificar o relato da doença com maior frequência em mulheres de 41 a 55 anos e a predisposição genética em parentes de primeiro grau em 55,25\% dos entrevistados. Os resultados obtidos de DM2 autorreferida de 23,68\% foram ainda maiores que os divulgados pela Sociedade Brasileira de Diabetes ${ }^{10}$ (SBD) em 2019 (7,9\% de prevalência) mas estão em concordância quando compara-se o gênero analisado, tal que demonstra que o diabetes acomete mais mulheres. A prevalência do acometimento a mulheres de DM2 não é consensual nos estudos, quan- do separados por gênero pela $\mathrm{SBD}^{10} \mathrm{em}$ diagnosticados por Teste Oral de Tolerância à Glicose identifica-se a prevalência dominante no sexo masculino ${ }^{10}$. Por se tratar de autorrelato, pode-se estar relacionado pela maior procura de serviços de serviços de saúde por parte delas ${ }^{17}$. Quanto a idade prevalente identificada de 41 a 55 anos em mulheres cuidadoras é colocada em comparação com os dados da SBD10, identifica-se que a idade está em divergência pois a prevalência da doença nesta base refere-se a 65 anos ou acima.

As alterações nos pés demonstram o autocuidado ineficaz dos cuidadores que podem levar a graves consequências a estes indivíduos ${ }^{18}$, entre os achados da inspeção nos pés de cuidadores diabéticos autorreferidos seguindo o instrumento do Protocolo de Cuidado com os Pés de Pessoas com Formulário para Avaliação de Neuropatia e Doença Arterial Periférica -Ambulatório de Especialidade ${ }^{14}$ destacam-se: pele ressecada e fissuras (48\%), calosidades (40\%), micose ungueal ou digital (37\%) seguidos de cor de pele anormal
$(7,4 \%)$, vasos dilatados dorsais e edemas (11\%). Os escores entrados nos cuidado-

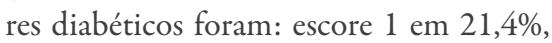
escore 2 em 7,1\%, escore 3 a 5 em 14\% que necessitam de avaliação periódica e aprazamento entre as consultas de um a doze meses $^{19}$. A prevalência de pele ressecada e fissuras pode ser justificada pelo ressecamento tornar a pele suscetível a fissuras, levando a perda da barreira protetora da pele e torna-a mais suscetível a entrada de microorganismos, resultando em quadros infecciosos. Embora não tenha achados de úlceras nos participantes, a prevenção é essencial pois $85 \%$ das amputações em diabéticos são decorrentes de úlceras ${ }^{19}$.

Estima-se que no Brasil existam cerca de 200 mil cuidadores que necessitam esclarecer suas dúvidas e expor suas angústias, pois, dessa forma, é possível a elaboração de ações que o orientem melhor nos cuidados com o paciente ${ }^{19}$. Evidencia-se a importância e necessidade de os profissionais de saúde orientarem os cuidadores quanto as práticas de inspeção dos pés com a transmissão de informações de for-

\section{Quadro I - Enumeração dos resultados obtidos através das questões apresentadas na metodologia.}

\begin{tabular}{|c|c|c|c|}
\hline QUESTÕES & RESPOSTA MÉDIA & N & $\%$ \\
\hline Você possui diabetes mellitus tipo 2? & Não & 87 & $76,32 \%$ \\
\hline Se sim, há quanto tempo? & 2a 3 anos & 15 & $13,16 \%$ \\
\hline Tem mais alguma pessoa na sua casa que é diabética(o)? & Sim & 56 & $49,12 \%$ \\
\hline Na sua família tem alguém diabético? & Sim & 73 & $64,04 \%$ \\
\hline Qual o grau de parentesco? & 1 ${ }^{\circ}$ Grau (pais e/ou mães) & 63 & $55,26 \%$ \\
\hline Fonte: Sousa TL, Costa cnN. unIP, SP. 2020.
\end{tabular}

Quadro 2 - Portadores de Diabetes Mellitus separados gênero e idade indicando quantidade, percentual e total. Santana de Parnaíba, São Paulo, Brasil, 2019.

\begin{tabular}{|c|c|c|c|}
\hline GÊNERO DOS CUIDADORES DIABÉTICOS & IDADE & N & $\%$ \\
\hline \multirow{3}{*}{ Feminino } & 25 a 40 anos & 6 & $22,22 \%$ \\
\cline { 2 - 4 } & 41 a 55 anos & 9 & $33,33 \%$ \\
\cline { 2 - 4 } & 56 a 70 anos & 6 & $22,22 \%$ \\
\hline \multirow{2}{*}{ Masculino } & 25 a 40 anos & 2 & $7,41 \%$ \\
\cline { 2 - 4 } & 41 a 55 anos & 3 & $11,11 \%$ \\
\cline { 2 - 4 } & 56 a 70 anos & 1 & $3,70 \%$ \\
\hline Total & & 27 & $100 \%$ \\
\hline Fonte: Sousa TL, Costa CNN. UNIP, SP. 2020. & \multicolumn{2}{|c}{} \\
\hline
\end{tabular}


ma acessível que envolvam demonstração e supervisão das práticas ${ }^{18}$. Deve-se sugerir a adesão de cuidados com os pés, incluindo a inspeção diária, hidratar a pele, cortar as unhas e não remover calos, entre outros específicos, como a procura de um profissional caso encontrem fissuras, bolhas, úlceras, micose interdigital, edema, hiperemia e onicomicoses ${ }^{19}$. Como prioridade, a Estratégia Saúde da Família é uma das responsáveis pelas ações de promover, proteger e recuperar a saúde ${ }^{18}$.

Salienta-se que este estudo teve por foco a prevalência do DM2 e a inspeção dos pés nos indivíduos cuidadores com diabetes autorreferidos, desta forma os resultados revelam uma problemática de uma realidade local e em uma única instituição, os dados não são generalizáveis. Sugere-se novas pesquisas com mais insti-

tuições para avaliar de forma mais ampla esta temática preocupante.

\section{Como prioridade,}

a Estratégia

Saúde da

Família é

uma das responsáveis

pelas ações de

promover, proteger

e recuperar a saúde.

\section{CONCLUSÕES}

Este estudo permitiu a avaliação dos pés diabéticos e a identificação da prevalência da DM2 em cuidadores de crianças e adolescentes com deficiências múltiplas de uma associação filantrópica através do questionário. Verificou-se a prevalência preocupante da doença e diversas alterações nos pés dos diabéticos. Neste contexto, a proposta sugerida deste estudo consiste na ampliação do cuidado da instituição para além dos pacientes atendidos, incluindo também seus cuidadores. Há uma grande necessidade da intervenção por meio de ações em estratégias municipais com foco na atenção primária e estratégia de saúde da família.

\section{REFERÊNCIAS}

1 SBD. Sociedade Brasileira de Diabetes. Diretrizes da Sociedade Brasileira de Diabetes: 2019-2020. São Paulo: Clannad; 2020.

2 Viana CSA, Salomão BT, Olivon VC, Pereira FAL, Viana CJ. Avaliação do risco para desenvolvimento de diabetes mellitus tipo 2 em estudantes universitários. Rev. Ciênc. Plural. 2019;5(2):94-110.

3 Soares RL, Ribeiro SMO, Fachin LB, Lima ACTS, Ramos LO, Ferreira LV. Avaliação de rotina do pé diabético em pacientes internados - prevalência de neuropatia e vasculopatia. FU Rev.2018;43(3):205-10.

4 Wilson HS. Family caregiving for a relative with Alzheimer's dementia: coping with negative choices. Nursing Research. 1989;38(2):94-98.

5 Vale JMM, Marques NAC, Santos LMS, Santana ME. Autocuidado do cuidador de adoecidos em cuidados paliativos oncológicos. Rev. Enf. UFPE. 2019;13(1)1-11.

6 Orem DE. Nursing: concepts of practice. $6^{\circ}$ ed. New York: Mosby; 2001.

7 Costa SRD, Castro EAB. Autocuidado do cuidador familiar de adultos ou idosos dependentes após a alta hospitalar. Rev. bras. enferm. 2014;67(6):979-986.

8 Reis MS, Silva RDS, Amorim MLC, Lopes KAT, Corrêa LS, Leão DP. Avaliação da qualidade de vida de cuidadores de pessoas com deficiência física e intelectual: um estudo com Whoqol-Bref. Rev. Bras. de prescrição e fisiologia do exercício. 2017;11(67):399-04.

9 Bertonhi LG, Dias JCR. Diabetes mellitus tipo 2: aspectos clínicos, tratamento e conduta dietoterápica. Revista Ciências Nutricionais Online. 2018;2(2)1-10.

10 SBD - Sociedade Brasileira de Diabetes. Dados epidemiológicos do diabetes mellitus no Brasil: 2018-2019. São Paulo: Clannad; 2019.

11 Varela DL. Estudo de Condução Nervosa dos Nervos Sural Dorsal e Plantar Medial no Diagnóstico Precoce De Neuropatia Diabética [dissertação]. Passo fundo: Universidade de Passo Fundo;2016.

12 Santos AL, Marcon SS, Silva SS, Teston EF, Back IR, Lino IGT et all. Adesão ao tratamento de diabetes Mellitus e relação com a assistência na atenção primária. Revista Mineira de Enfermagem. 2020;24(1)1-10.

13 Santos EQN. Principais cuidados de enfermagem na prevenção do Diabetes Mellitus(DM): foco na gestão em saúde [tese]. São Francisco do Conde: Instituto de Educação a Distância, Universidade da Integração Internacional da Lusofonia Afro-Brasileira;2019.

14 Governo Do Distrito Federal; Secretaria De Estado De Saúde; Subsecretaria De Atenção Integral À Saúde; Comissão Permanente De Protocolos De Atenção À Saúde. Protocolo de Cuidado com os Pés de Pessoas com Diabetes Mellitus na SES/DF. Portaria SES-DF Nº 1356 de 05/12/2018. 2018;1(1)1-30

15 Conselho Nacional Da Saúde. Resolução $n^{\circ}$ 466, de dezembro de 2012. Aprova as seguintes diretrizes e normas regulamentadoras de pesquisas envolvendo seres humanos. 2013.

16 Bardin L. Análise de conteúdo. Lisboa: Edições; 2009.

17 Iser BPM, Stope SR, Chueiri OS, Szwarcwald CL, Malta DC, Monteiro HOC. Prevalência de diabetes autorreferido no Brasil: resultados da Pesquisa Nacional de Saúde 2013. Epidemiol. Serv. Saúde[2237-9622]. 2015Apr-jun [Cited 2020 oct 20];24(2)305-314. Available from: https://www.scielosp.org/ article/ress/2015.v24n2/305-314/pt 